\title{
Comparison of Echinococcus multilocularis and Echinococcus granulosus hydatid fluid proteome provides molecular strategies for specialized host-parasite interactions
}

\author{
Chun-Seob Ahn ${ }^{1}$, Jeong-Geun Kim ${ }^{1}$, Xiumin Han ${ }^{2,3}$, Insug Kang ${ }^{4}$ and Yoon Kong ${ }^{1}$ \\ ${ }^{1}$ Department of Molecular Parasitology, Samsung Medical Center, Sungkyunkwan University School of Medicine, Suwon, \\ Korea \\ ${ }^{2}$ Qinghai Province Institute for Endemic Diseases Prevention and Control, Xining, China \\ ${ }^{3}$ Clinical Research Institute for Hydatid Disease, Qinghai Provincial People's Hospital, Xining, China \\ ${ }^{4}$ Department of Molecular Biology and Biochemistry, Kyung Hee University School of Medicine, Seoul, Korea \\ Correspondence to: Yoon Kong, email: kongy@skku.edu \\ Keywords: alveolar echinococcosis, cystic echinococcosis, hydatid fluid, proteome, protein-protein interaction networks \\ Received: June 22, $2017 \quad$ Accepted: August 09, $2017 \quad$ Published: September 08, 2017 \\ Copyright: Ahn et al. This is an open-access article distributed under the terms of the Creative Commons Attribution License 3.0 \\ (CC BY 3.0), which permits unrestricted use, distribution, and reproduction in any medium, provided the original author and source \\ are credited.
}

\section{ABSTRACT}

\begin{abstract}
Alveolar and cystic echinococcoses, caused by the metacestodes of Echinococcus multilocularis and E. granulosus, are prevalent in several regions and invoke deleterious zoonotic helminthiases. Hydatid fluid (HF), which contains proteinaceous and non-proteinaceous secretions of the parasite- and host-derived components, critically affects the host-parasite interplay and disease progression. We conducted HF proteome profiling of fully mature $E$. multilocularis vesicle (nine months postinfection) and $E$. granulosus cyst (stage 2). We identified 120 and 153 proteins, respectively, in each fluid. Fifty-six and 84 proteins represented distinct species; 44 and 66 were parasites, and 12 and 18 were host-derived proteins. The five major parasite protein populations, which included antigen B isoforms, metabolic enzymes, proteases and inhibitors, extracellular matrix molecules (ECMs), and developmental proteins, were abundantly distributed in both fluids and also exclusively in one sample or the other. Carbohydrate-metabolizing enzymes were enriched in $E$. granulosus HF. In the $E$. multilocularis HF, proteins that constitute ECMs, which might facilitate adhesion and cytogenesis, were highly expressed. Those molecules had physical and functional relationships along with their biochemical properties through protein-protein interaction networks. Twelve host-derived proteins were largely segregated to serum components. The major proteins commonly and uniquely detected in these HFs and their symbiotic interactome relationships might reflect their biological roles in similar but distinct modes of maturation, invasion, and the longevity of the parasites in the hosts.
\end{abstract}

\section{INTRODUCTION}

Echinococcosis refers to a disease complex caused by the metacestodes of the genus Echinococcus. More than seven Echinococcus spp., such as E. granulosus (G1), E. ortleppi (G4), E. canadensis (G6, 7, 8, and 10), E. oligathus, E. vogeli, and E. multilocularis, trigger human infections, among which E. granulosus and $E$. multilocularis are the most important pathogenic species [1]. Humans serve as intermediate hosts and are infected with the larval stage of the worms. When humans incidentally ingest parasite eggs, oncospheres hatched out from the eggs are activated in the small intestine. The oncospheres are released into the bloodstream and mostly end up lodged in the liver. The parasites grow into unilocular and multilocular cystic masses that result in 
cystic echinococcosis (CE) and alveolar echinococcosis (AE) [1].

$\mathrm{CE}$ is prevalent in nomadic areas in association with dog-rearing environments [2]. AE is increasingly detected in high-altitude forested and pastoral areas in the Northern Hemisphere, which includes enclaves in Europe, central Asia, and northwestern China [3, 4]. CE and $\mathrm{AE}$ are the most deleterious enzootic diseases and have a great impact on disability-adjusted life years [4, 5]. These larval cestodiases exemplify the top-ranking entities among the neglected tropical diseases by the World Health Organization due to their significant disease burden and associated socioeconomic losses (http://who. int/neglected_diseases/diseases/en/).

Echinococcus granulosus and E. multilocularis are the nearest phylogenetic neighbors in the family Taeniidae [6]: the two organisms share multiple aspects of growth and developmental plasticity and similarities in their intermediate and definitive hosts. The genomes of these two parasites each comprise approximately 115 megabases and show $96 \%$ sequence identity within the coding regions $[7,8]$. They display the unique expansion of similar gene families that specialize in nutrient uptake from the host and protection from host defensive system [8]. Interestingly, they differ markedly in their biological features, such as morphological characteristics during growth and development, and invasion into adjacent tissues within the intermediate hosts. E. granulosus metacestode grows slowly to form unilocular cyst that expand and crowd the affected organs and tissues; conversely, $E$. multilocularis metacestode forms multivesiculated cystic mass and shows peripheral infiltration with central necrosis, which resembles invasive malignant tumors [1, 9]. The intermediate hosts of the parasites differ, ungulates and humans for $\mathrm{CE}$ and rodents and humans for $\mathrm{AE}$.

The hydatid cyst has three major components: the germinal layer, the protoscolex, and hydatid fluid (HF). The germinal layer constitutes the outermost morphological contour and is responsible for the uptake of essential resources from the host through the syncytial membrane. The protoscolex asexually buds from the brood capsule, which protrudes from the germinal layer, and develops into an adult when it infects the definitive host $[1,5]$. HF comprises numerous proteinaceous and non-proteinaceous materials, which are secreted from the parasites as well as absorbed from the host [10]. HF also harbors tegumental materials shed from the protoscolex and resources derived from metabolic turnover of the germinal layer $[11,12]$. HF proteins are crucial at the host-parasite interface for maturation and survival of the parasites within the host and disease progression $[12,13]$. The major biological functions of the HF proteins include activating and inhibiting immune and inflammatory cells and inducing Th2-biased host immune responses [11, 14]. The HF proteins participate in transportation and assimilation of host essential lipids, thus contributing to the long-term survival of the parasites [15]. The proteins also have protective functions that include antioxidant activity against oxidative stresses and proteolytic enzymes responsible for defense from host immune attacks $[16,17]$.

A number of studies have focused on identifying the global protein profile and biological reactivity of $E$. granulosus HF (EgHF) [11-13], but no study has yet analyzed the functional relationships and molecular interactions of EgHF proteins. The proteome profile of E. multilocularis HF (EmHF) was recently addressed during expressional analyses of $E$. multilocularis antigen $\mathrm{B}$ (EmAgB) isoforms [15]. However, comprehensive investigations and systematic analyses of EmHF regarding their biochemical and biological properties have not been conducted. More importantly, comparative data on E. granulosus and E. multilocularis HF proteomes are scarce. AE mass and CE cyst share a variety of biological similarities, but simultaneously, they show significant differences from each other. The HF from these closely related organisms might contain either unique or common proteins that are germane to their specialized biological features.

In the present study, we comparatively analyzed the proteome profiles of EmHF and EgHF obtained from experimentally or naturally infected hosts. HFs collected from immunologically competent hosts might reflect the genuine nature of HF proteomes [15]. Our data strongly suggest a possible biological function of HF proteins that could be correlated to the distinctive morphology, development, and longevity of parasites in hosts.

\section{RESULTS}

\section{Proteome analysis of EmHF and EgHF grown in immunocompetent hosts}

Figure 1A displays the protein profile of fully mature EmHF (9 months) and EgHF (CE2 cyst) separated by $10 \%$ Tricine SDS-PAGE under reducing conditions. Comparative analyses of the both fluids revealed similar but distinct protein banding patterns. More than 50 protein bands, whose molecular weights ranged from below 6 $\mathrm{kDa}$ to over $200 \mathrm{kDa}$, were typically observed. The EmHF exhibited prominent bands at $6,9,16$, and $64 \mathrm{kDa}$ in addition to high molecular proteins $>72 \mathrm{kDa}$. The EgHF showed noticeable bands at 8, 14, 22, 34, and $64 \mathrm{kDa}$. High molecular proteins $>75 \mathrm{kDa}$ were also deeply stained. In general, more prominent and clear banding patterns were evident in the EgHF compared with in the EmHF.

The protein bands were sliced into 22 gel pieces along with their molecular weights, tryptic digested, and analyzed through nano-liquid chromatographyelectrospray ionization-multi-stage mass spectrometry (LC-ESI-MS/MS). We were able to identify 120 proteins from the EmHF, of which $85(70.8 \%)$ and 35 proteins $(29.2 \%)$ originated from the parasite and host, respectively. 
Of these proteins, $56(46.7 \%)$ represented distinctive protein species; 44 parasite $(36.7 \%)$ and 12 host proteins (10\%) (Figures 1B and 1C, and Supplementary Table 1). The major protein fractions derived from the parasite constituted with $13 \mathrm{EmAgB}$ isoforms (one EmAgB1, two EmAgB2, three EmAgB3a, four EmAgB3b/c, and three EmAgB4), nine $\mathrm{N}$-acetylated $\alpha$-linked acidic dipeptidase 2 (NAALAD2), each of six $\alpha 2$ macroglobulin and low-density lipoprotein receptor (LDLR), and three prosaposin (Table 1). We differentially classified $\mathrm{EmAgB} 3$ proteins as EmAgB3a and EmAgB3b/c because these molecules were synthesized from different genes: EmAgB3a from EmuJ_000381500 and EmAgB3b/c from EmuJ_000381600 and/or EmuJ_000381700 [17]. We also detected a number of ECM-associated proteins, such as different types of collagen $\alpha 1$ (IV and V), collagen XI $\alpha 2$, and fibrillar collagen FAp $1 \alpha$. Host proteins were largely allocated into serum components, specifically, albumin (15 entries) and immunoglobulin (three molecules) (Supplementary Table 1).

We detected 153 proteins (132 parasite [86.3\%] and 21 host proteins [13.7\%]), among which 84 entities $(54.9 \%)$ were distinct proteins (66 parasite [43.1\%] and 18 host proteins [11.8\%]) in the EgHF. A total of 42 entries of diverse $\mathrm{EgAgB}$ isoforms comprised 11 each of $\mathrm{EgAgB} 1$ and $\mathrm{EgAgB} 2$, six EgAgB3, and $14 \mathrm{EgAgB} 4$. In addition, eight glycoprotein antigen 5, five peptidase inhibitor 16 , and four gynecophoral canal proteins (fasciclin) [18] consisted of the major parasite protein fractions (Table 2). We found abundant serum components including serotransferrin and albumin as host-derived molecules (Supplementary Table 2).

Overall, LC-MS/MS analysis identified 82 parasite proteins, with 16 EmHF proteins, $38 \mathrm{EgHF}$ proteins, and
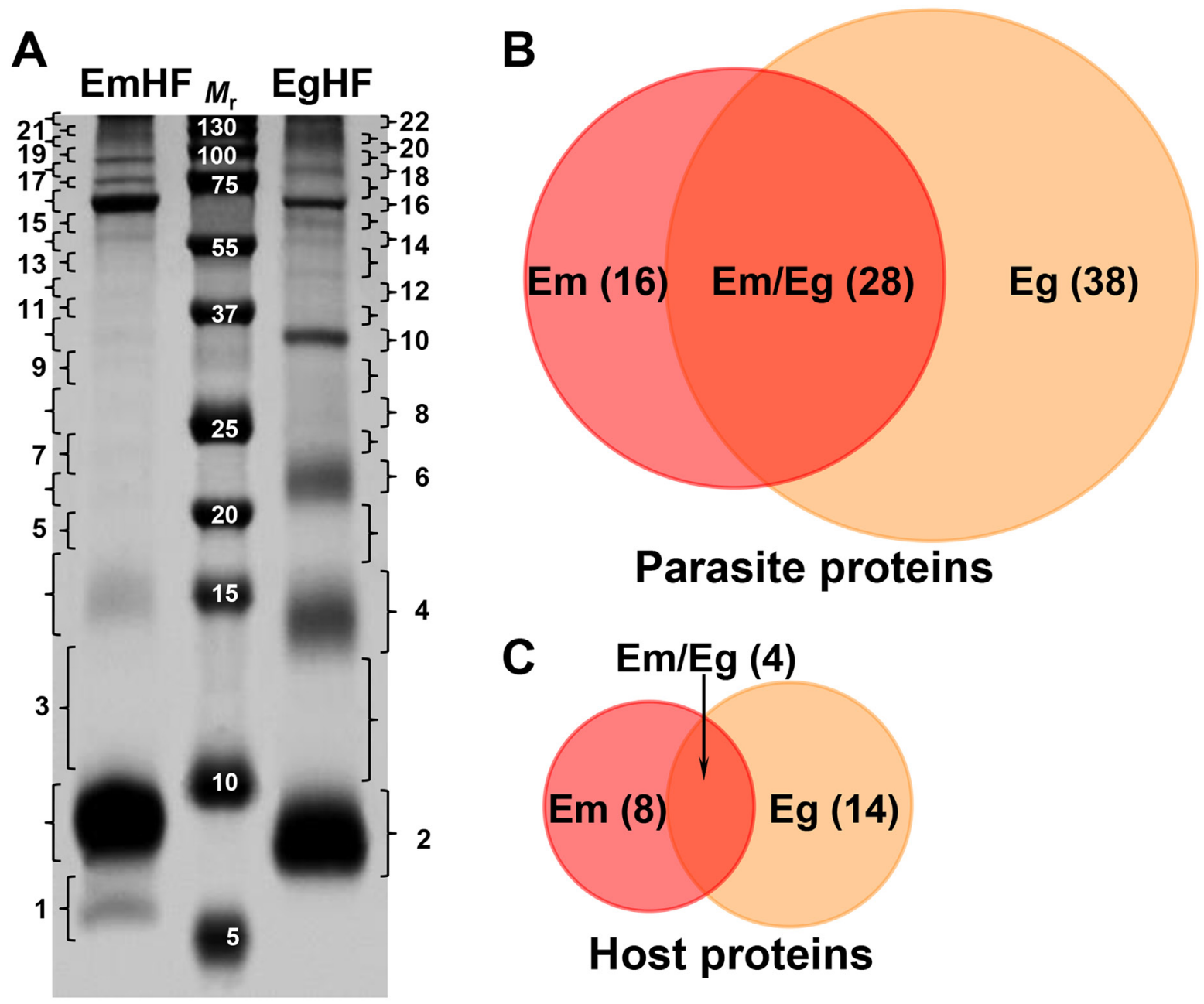

Figure 1: Electrophoretic profile and protein identification of the hydatid fluid of $E$. multilocularis (EmHF) and E. granulosus (EgHF). (A) EmHF and EgHF (each $20 \mu \mathrm{g}$ ) were separated by 10\% Tricine SDS-PAGE under reducing conditions. The protein bands were divided into 22 pieces and processed with in-gel tryptic digestion prior to LC-ESI-MS/MS analysis. $M_{\mathrm{r}}$, molecular weight in $\mathrm{kDa}$. (B and C) Comparison of protein components identified in the Echinococcus HF samples by Venn diagrams. Parasite proteins (B) and host-derived proteins (C). 
Table 1: Parasite proteins exclusively recognized in EmHF

\begin{tabular}{|c|c|c|c|c|c|c|}
\hline $\begin{array}{l}\text { Serial } \\
\text { No. }\end{array}$ & Band nos. & Sum of emPAI ${ }^{1}$ & Description & $\begin{array}{c}\text { Accession no. }{ }^{2} \\
(\text { EmuJ_ })\end{array}$ & $\begin{array}{c}\text { Mouse } \\
\text { homolog }^{3}\end{array}$ & $\begin{array}{l}\text { Putative } \\
\text { function }\end{array}$ \\
\hline 1 & 4 & 0.49 & FABP & 000550000 & P04117 & Transporter \\
\hline 2 & 4 & 0.49 & Hedgehog & 001200300 & Q61488 & Development \\
\hline 3 & 4 & 0.49 & $\begin{array}{l}\text { Zinc finger, } \\
\mathrm{C} 2 \mathrm{H} 2\end{array}$ & 000194800 & Q03267 & Stress response \\
\hline 4 & 7 & 0.13 & Collagen XI $\alpha 2$ & 000524200 & Q64739 & ECM \\
\hline 5 & 7 & 0.13 & GST & 000538300 & P48774 & Antioxidant \\
\hline 6 & 8 & 0.04 & FGFR & 000842900 & Q9QWY8 & $\begin{array}{c}\text { Cellular } \\
\text { interaction }\end{array}$ \\
\hline 7 & $10,11,15,17-22$ & 2.45 & NAALAD2 & 000908900 & Q9CZR2 & Protease \\
\hline 8 & $\begin{array}{c}10,11,13,18 \\
19,21,22\end{array}$ & 0.99 & $\alpha 2$ macroglobulin & 000641100 & Q6GQT1 & $\begin{array}{l}\text { Binding activity, } \\
\text { Protease inhibitor }\end{array}$ \\
\hline 9 & 11 & 0.08 & Annexin & 000193700 & P10107 & Binding activity \\
\hline 10 & $12,17,18,20-22$ & 0.89 & LDLR & 000701800 & O88572 & Signaling \\
\hline 11 & 17 & 0.1 & Gp50 & 001201600 & - & Th2 response \\
\hline 12 & 17 & 0.1 & CHP & 001058700 & - & Not-available \\
\hline 13 & 18 & 0.03 & OTK & 000212300 & Q9QVP9 & Kinase \\
\hline 14 & 19 & 0.02 & Collagen $\alpha 2(\mathrm{I})$ & 000823800 & Q64739 & $\mathrm{ECM}$ \\
\hline 15 & 21 & 0.03 & NAGAB & 000716600 & Q8BHN3 & $\begin{array}{c}\text { Carbohydrate } \\
\text { metabolism }\end{array}$ \\
\hline 16 & 21,22 & 0.11 & Collagen $\alpha 1(\mathrm{~V})$ & 000140100 & O88207 & $\mathrm{ECM}$ \\
\hline
\end{tabular}

${ }^{1}$ Exponentially modified protein abundance index.

${ }^{2}$ Accession numbers were obtained from NCBInr DB (http://www.ncbi.nlm.nih.gov/) and E. multilocularis DB (http://www. genedb.org/Homepage/Emultilocularis).

${ }^{3}$ Mouse homologs were retrieved using Uniprot software (http://www.uniprot.org/).

CHP, conserved hypothetical protein; FABP, fatty acid binding protein; FGFR, fibroblastic growth factor receptor; GST, glutathione transferase; Gp50, diagnostic antigen gp50; LDLR, low density lipoprotein receptor; NAALAD2, N-acetylated $\alpha$-linked acidic dipeptidase 2; NAGAB, neutral $\alpha$ glucosidase AB; OTK, tyrosine protein kinase otk.

28 proteins shared between EmHF and EgHF (Figure 1B, and Tables 1-3). We identified 26 different host-derived proteins in the EmHF and EgHF; four proteins were common to both fluids, whereas we detected eight only in EmHF and 14 proteins exclusively in EgHF (Figure $1 \mathrm{C}$, and Supplementary Table 3 and Supplementary Table 4). These results suggested the close phylogenetic and evolutionary relationships but also distinct biological behaviors of these two parasites within their host environments.

\section{Gene ontology (GO) terms assigned to proteins identified in EmHF and EgHF}

The systematic analysis employing GO terms were assigned to the identified proteins on the basis of similarity patterns by Blast2GO in the second level subcategories [19]. Figure 2 presents the distribution patterns of the parasite proteins along with their given ontological terms. The proteins associated with biological process revealed somewhat dissimilar distribution patterns. Molecules related to metabolic process, cellular process, single-organism process, and biological regulatory molecules were commonly detected in both fluids. We also recognized proteins involved in developmental and multicellular organism processes, localization, and signaling in both samples. However, molecules associated with the immune and protective system, biogenesis, and adhesion were observed exclusively in EgHF. In the molecular function and cellular component subcategories, relatively large proportions of the proteins were shared by both parasites, but those related to molecular function 
Table 2: Parasite proteins observed uniquely in EgHF

\begin{tabular}{|c|c|c|c|c|c|c|}
\hline $\begin{array}{l}\text { Serial } \\
\text { No. }\end{array}$ & $\begin{array}{l}\text { Band } \\
\text { nos. }\end{array}$ & $\begin{array}{l}\text { Sum of } \\
\text { emPAI }\end{array}$ & Description & $\begin{array}{l}\text { Accession no. }{ }^{2} \\
\text { (EgrG__ }\end{array}$ & Mouse homolog ${ }^{3}$ & Principal functions \\
\hline 1 & $3-5$ & 0.63 & ECP & 000378300 & - & Not-available \\
\hline 2 & 3 & 0.28 & ECP & 000956500 & - & Not-available \\
\hline 3 & 3 & 0.22 & SOD1 & 000638300 & P08228 & Antioxidant \\
\hline 4 & 3 & 0.26 & Profilin & 000122100 & - & Actin binding \\
\hline 5 & 4 & 1.86 & $\mathrm{ECP}$ & 000079500 & - & Not-available \\
\hline 6 & 5,6 & 1.62 & Ferritin & 000382200 & P09528 & Iron transport \\
\hline 7 & 5 & 0.2 & snRNP & 000525910 & - & Isomerase \\
\hline 8 & 6 & 2.08 & $\mathrm{ECP}$ & 000596300 & - & Not-available \\
\hline 9 & 6 & 1.29 & ECP & 000315600 & - & Not-available \\
\hline 10 & 6 & 0.35 & ThioTrx & 000791700 & P35700 & Redox homeostasis \\
\hline 11 & $6-10$ & 1.42 & PI16 & 000766600 & P49935 & Protease inhibitor \\
\hline 12 & 7 & 0.16 & Niemann Pick & 000682900 & Q9Z0J0 & Lipid metabolism \\
\hline 13 & 7 & 0.13 & Complement TNF & 001189200 & Q8R2Z0 & $\begin{array}{l}\text { Carbohydrate } \\
\text { metabolism }\end{array}$ \\
\hline 14 & 7 & 0.12 & TPI & 000416400 & P17751 & $\begin{array}{l}\text { Carbohydrate } \\
\text { metabolism }\end{array}$ \\
\hline 15 & 8 & 0.57 & $14-3-3$ protein & 000789700 & P16054 & Signaling \\
\hline 16 & 8 & 0.26 & IoMT & 001133400 & P23506 & Transferase \\
\hline 17 & 8 & 0.1 & PGM & 000799500 & O70250 & $\begin{array}{l}\text { Carbohydrate } \\
\text { metabolism }\end{array}$ \\
\hline 18 & 10,11 & 1.52 & $\mathrm{MDH}$ & 001185000 & P08249 & $\begin{array}{l}\text { Carbohydrate } \\
\text { metabolism }\end{array}$ \\
\hline 19 & 10 & 0.31 & LDHA & 000660800 & P06151 & $\begin{array}{l}\text { Carbohydrate } \\
\text { metabolism }\end{array}$ \\
\hline 20 & 10,11 & 0.4 & Succinyl CoA syn & 001199000 & Q9Z2I8 & Ligase \\
\hline 21 & 10 & 0.23 & IGBP & 000799300 & P05017 & Signaling \\
\hline 22 & 10 & 0.29 & Transaldolase & 000092800 & Q93092 & $\begin{array}{c}\text { Carbohydrate } \\
\text { metabolism }\end{array}$ \\
\hline 23 & 10 & 0.08 & $\mathrm{PDH}$ & 000956200 & O70571 & $\begin{array}{l}\text { Carbohydrate } \\
\text { metabolism }\end{array}$ \\
\hline 24 & 12 & 1.15 & OA & 001032200 & P29758 & Transferase \\
\hline 25 & 13 & 1.54 & Citrate synthase & 001028500 & Q9CZU6 & $\begin{array}{l}\text { Carbohydrate } \\
\text { metabolism }\end{array}$ \\
\hline 26 & 13 & 0.4 & NADPH & 001068500 & P54071 & $\begin{array}{l}\text { Carbohydrate } \\
\text { metabolism }\end{array}$ \\
\hline 27 & 13 & 0.25 & PDHE1 & 000590700 & P35486 & $\begin{array}{l}\text { Carbohydrate } \\
\text { metabolism }\end{array}$ \\
\hline 28 & 13 & 0.12 & Acyl CoA HT & 001087900 & Q9DBK0 & Lipid metabolism \\
\hline 29 & 14,15 & 1.21 & Calnexin & 000875100 & P35564 & Chaperone \\
\hline 30 & 14 & 0.27 & Iron:zinc AP & 001169400 & Q8BX37 & Hydrolase \\
\hline
\end{tabular}

(Continued) 


\begin{tabular}{|c|c|c|c|c|c|c|}
\hline $\begin{array}{l}\text { Serial } \\
\text { No. }\end{array}$ & $\begin{array}{l}\text { Band } \\
\text { nos. }\end{array}$ & $\begin{array}{l}\text { Sum of } \\
\text { emPAI }{ }^{1}\end{array}$ & Description & $\begin{array}{l}\text { Accession no. }{ }^{2} \\
\text { (EgrG__ }\end{array}$ & Mouse homolog ${ }^{3}$ & Principal functions \\
\hline 31 & 14 & 0.12 & GD & 000589100 & P26443 & Oxidoreductase \\
\hline 32 & 16 & 1.33 & VAT & 000317300 & Q8BRU6 & Transport \\
\hline 33 & 16 & 0.06 & Lysyl oxidase & 000217900 & P58022 & Oxidoreductase \\
\hline 34 & 16 & 0.12 & AspRNA & 000777100 & Q922B2 & Protein biosynthesis \\
\hline 35 & 17,19 & 1.21 & PEPCK & 000292700 & Q8BH04 & $\begin{array}{c}\text { Carbohydrate } \\
\text { metabolism }\end{array}$ \\
\hline 36 & 20 & 0.07 & Macroglobulin & 000641200 & Q3UU35 & $\begin{array}{l}\text { Serine protease } \\
\text { inhibitor }\end{array}$ \\
\hline 37 & 21 & 0.05 & Collagen $\alpha 1(\mathrm{XV})$ & 000729300 & O35206 & ECM \\
\hline 38 & 22 & 0.04 & Hemicentin 1 & 000422350 & D3YXG0 & Cell cycle \\
\hline
\end{tabular}

${ }^{1}$ Exponentially modified protein abundance index.

${ }^{2}$ Accession numbers were obtained from NCBInr DB (http://www.ncbi.nlm.nih.gov/) and E. granulosus DB (http://www. genedb.org/Homepage/Egranulosus).

${ }^{3}$ Mouse homologs were retrieved using Uniprot software (http://www.uniprot.org/).

Acyl CoA HT, acyl coenzyme A hydrolase transferase; AspRNA, aspartyl tRNA synthetase cytoplasmic; Complement

$\mathrm{TNF}$, complement $\mathrm{C} 1 \mathrm{q}$ tumor necrosis factor; ECP, expressed conserved protein; FABP, fatty acid binding protein;

FGFR, fibroblastic growth factor receptor; GD, glutamate dehydrogenase; GST, glutathione transferase; IoMT, protein

1 isoaspartate o methyltransferase; Iron:zinc AP, iron:zinc purple acid phosphatase; IGBP, insulin growth factor binding protein; LDHA, lactate dehydrogenase A; LDLR, low density lipoprotein receptor protein; MDH, malate dehydrogenase (mitochondrial); NAALAD2, N-acetylated alpha-linked acidic dipeptidase 2; NADPH, NADPH dependent isocitrate dehydrogenase; Niemann-Pick, Niemann Pick C2 protein; OA, ornithine aminotransferase; PDH, pyruvate dehydrogenase; PDHE1, PD E1 component subunit; PEPCK, phosphoenolpyruvate carboxykinase; PGM, phosphoglycerate mutase; PI16, peptidase inhibitor 16; snRNP, U snRNP cyclophilin; SOD1, superoxide dismutase 1 (soluble); Succinyl CoA syn, succinyl coenzyme A synthetase $\alpha$ subunit; TPI, triosephosphate isomerase; ThioTrx, thioredoxin peroxidase; VAT, vesicular amine transporter.

were more prominent in EmHF and those connected to cellular compartment were more enriched in EgHF.

Host proteins commonly and uniquely detected were largely grouped into metabolic process, cellular process, single-organism process, biological regulator and stimulus responses (biological process), and binders and catalysts (molecular function). The proteins linked to cellular component exhibited dissimilar distribution patterns along with the respective HF. Molecules located in membrane, extracellular regions, and organelle were relatively abundant in EmHF, whereas those associated with cellular and organelle structure were largely distributed in EgHF (Figure 3).

\section{Functional characterization of identified proteins}

The proteins identified in the EmHF and EgHF were further analyzed in accordance with their principal biochemical properties. We selected proteins that showed high abundance by emPAI values greater than 0.01 to ensure confidence in our protein identification and reproducibility. As shown in Figure 4A and Table 3, five major protein fractions were abundant in both fluids: i) several isoforms of antigen B molecules (AgB1-AgB4); ii) metabolic enzymes (phosphoglucose isomerase, fructose bisphosphate aldolase, enolase, malate dehydrogenase, glyceraldehyde 3-phosphate dehydrogenase, and lysosomal $\alpha$ mannosidase); iii) proteases and inhibitors (estrogen regulated protein 45, cathepsin B, glycoprotein antigen 5, ADAMTS protein 3 , and cystatin); iv) molecules associated with ECM and cytogenesis (gynecophoral canal protein, collagen, basement membrane-specific heparan sulfate, and laminin); and v) developmental and signaling proteins (abnormal EMBryolocus tagsis [embryogenesis family member] emb 9, neurogenic locus notch, and EGF domain protein).

$\mathrm{N}$-acetylated $\alpha$-linked acidic dipeptidase-like 2 (NAALADL2), which might be involved in adhesion and invasion through its proteolytic activity, and $\alpha 2$ macroglobulin, which inhibits protease activity, were uniquely and abundantly expressed in EmHF. We observed a signal-related molecule of LDLR only in EmHF (Table 1). In contrast, diverse enzymes involved in carbohydrate metabolism (citrate synthase, malate dehydrogenase, phosphoenolpyruvate carboxykinase, succinyl CoA synthetase, nicotineamide adenine dinucleotide phosphate reduced form, phosphopyuvate dehydrogenase E1, 


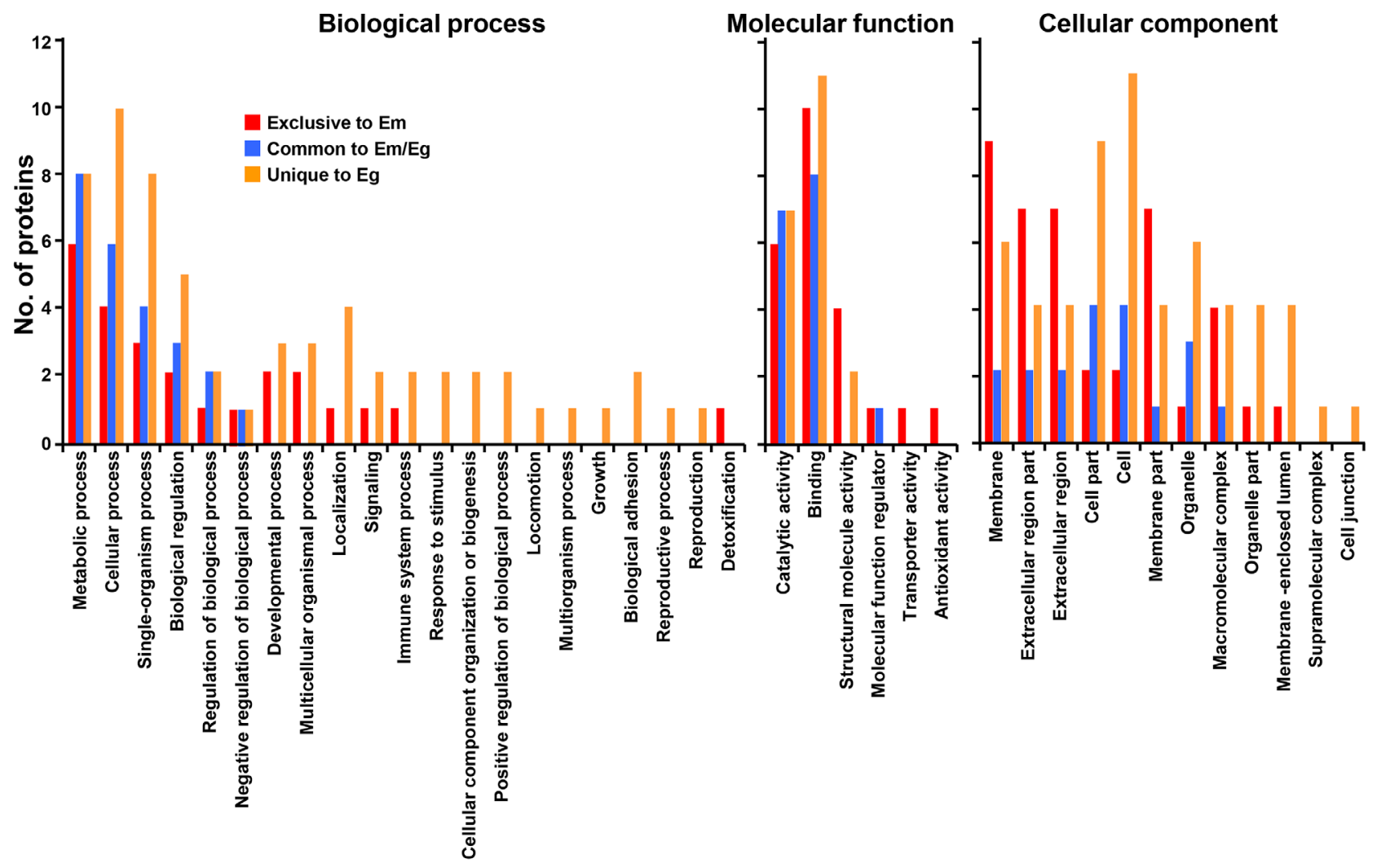

Figure 2: Classification of gene ontology of parasite proteins identified from E. multilocularis hydatid fluid (EmHF) and $\boldsymbol{E}$. granulosus HF (EgHF). The number of identified proteins in each functional group is shown in the histogram. The terms associated with the biological process, molecular function, and cellular component were adopted from Blast2GO based on similarity patterns using the second level of the GO hierarchy [19].

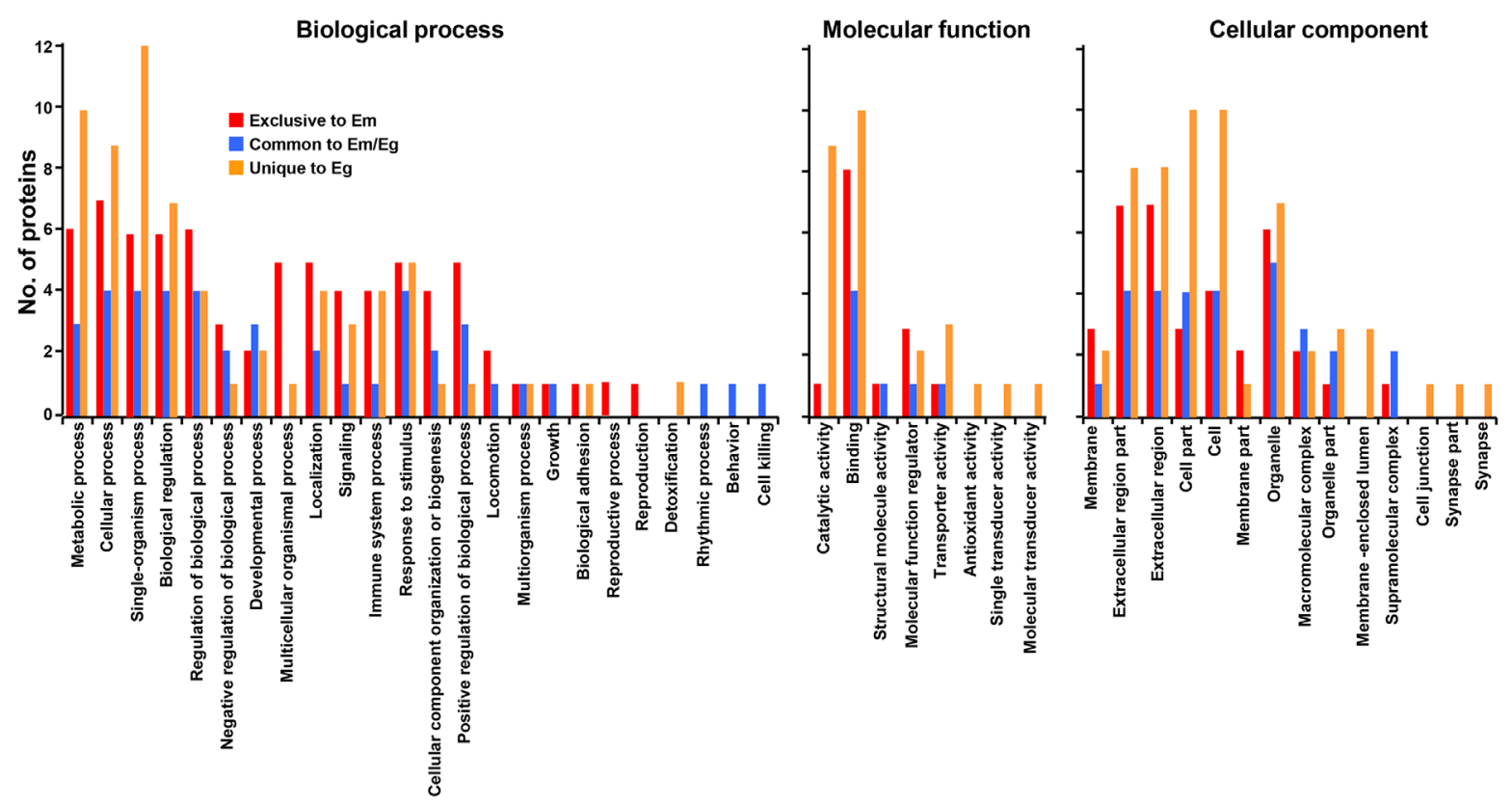

Figure 3: Analysis of gene ontology of host-derived proteins recognized in E. multilocularis hydatid fluid (EmHF) and E. granulosus HF (EgHF). The number of proteins in each functional group is shown by histogram. The terms assigned to the biological process, molecular function, and cellular component were tailored from Blast2GO based on similarities employing the second level of the GO hierarchy [19]. 
triosephosphate isomerase, and phosphoglycerate mutase) were exclusively detected in EgHF. We recognized antioxidant proteins (superoxide dismutase and thioredoxin peroxidase) and chaperone molecule (calnexin). In addition, signaling molecules (14-3-3 proteins and insulin-like growth factor-binding protein), ornithine aminotransferase, peptidase inhibitor 16, and three expressed conserved proteins (EgrG_000596300, EgrG_000079500, and EgrG_000315600) constituted relatively large fractions in EgHF (Table 2).

Major fractions of the host-derived molecules commonly found in both fluids were serum components, which included albumin, serotransferrin, $\alpha 1$ protease inhibitor (serpin), and immunoglobulins. We detected lipid- and carbohydrate-metabolizing enzymes exclusively in the EgHF proteome (Figure 4B, Supplementary Table 3 and Supplementary Table 4).

\section{Integrated network for protein-protein interactions (PPIs) in the HF proteomes}

Protein-protein interactions are critically involved in most cellular and biochemical processes. During proteome analysis of the EmHF and EgHF, we identified key molecules engaged in cytoskeletal and cytogenesis in EmHF, which included laminin, integrin, and a subset of diverse collagens. We also found considerable numbers of hub proteins, which importantly participated in carbohydrate metabolism in the EgHF (malate dehydrogenase 2, lactate dehydrogenase, enolase, transaldolase, and triosephosphate isomerase) (Tables 1-
3). Table 4 summarizes the numbers and functionality of the interacting proteins in the EmHF and EgHF identified through the STRING ver10 algorithm.

We analyzed the topology and centrality of the PPI network in the EmHF and EgHF to elucidate functional protein organization. Figure 5 depicts the integrated PPI network of the identified proteins. Numerous molecules interacted with one or more other proteins. One each of major functional cluster that showed a strong PPI relationship was evident along with their biochemical properties and/or biological roles. Of 66 proteins identified in the EgHF, $45(68.2 \%)$ revealed a complicated interaction network in which $24(36.4 \%)$ were tightly associated with carbohydrate metabolism (confined within the red dotted circle). Of 35 proteins in the EmHF, 24 (68.6\%) displayed interactome relationships. Molecules associated with ECM, adhesion, and cytogenesis (10 species; 28.6\%) demonstrated a strong functional network (Figure 5, marked by black dotted circle). The number of interacting proteins appeared to be larger than that of annotated proteins due to additional functionality of each molecule.

\section{DISCUSSION}

$\mathrm{AE}$ and $\mathrm{CE}$ metacestodes thrive for considerable periods in immunologically competent hosts and invoke significant illness. HF proteins continuously interact with the host as a first-line effector system to confront cytopathic environments [12-15, 20]. Their molecular interactions might critically affect parasite survival and subsequent disease progression. In this study, we

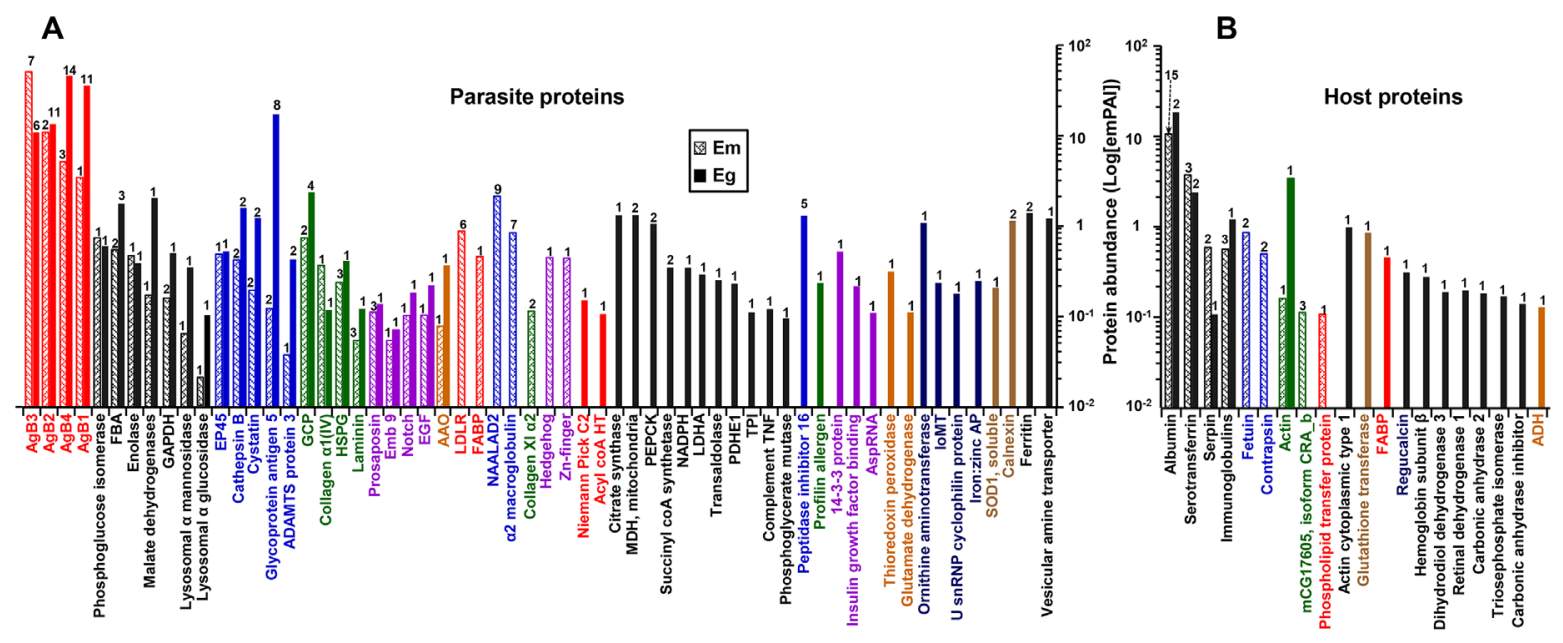

Figure 4: Differential heat map of the $E$. multilocularis hydatid fluid (EmHF) and E. granulosus HF (EgHF) proteins. The 26 most abundant parasite proteins (A) and top five host proteins (B) that showed emPAI values above 0.01 were analyzed. Protein abundance is presented by $\log [\mathrm{emPAI}]$. Antigen B isoforms, whose main functions are related to lipid transportation and assimilation, and Th2 responses are marked by red. Enzymes involved in carbohydrate metabolism are indicated by black. Molecules associated with proteolysis and inhibitors are shown in blue. Extracellular matrix and cytogenetic proteins are denoted by green. Molecules associated with cell growth and development are shown in purple. AgBs, Em and Eg antigen Bs; Ag5, glycoprotein antigen 5; AspRNA, aspartyl tRNA synthetase (cytoplasmic); GAPDH, glyceraldehyde 3-phosphate dehydrogenase; FBA, fructose bisphosphate aldolase; EP45, estrogen regulated protein 45; HSPG, basement membrane specific heparan sulfate; Emb9, abnormal EMBroylocus tagsis(embryogenesis family member) emb 9; Notch, neurogenic locus notch protein; EGF, EGF domain protein. 
Table 3: Parasite proteins identified in both EgHF and EmHF

\begin{tabular}{|c|c|c|c|c|c|c|}
\hline $\begin{array}{l}\text { Serial } \\
\text { No. }\end{array}$ & Band nos. & Sum of emPAI ${ }^{1}$ & Description & $\begin{array}{c}\text { Accession no. }{ }^{2} \\
\text { (EmuJ_/EgrG_) }\end{array}$ & $\begin{array}{c}\begin{array}{c}\text { Mouse } \\
\text { homolog }^{3}\end{array} \\
\end{array}$ & $\begin{array}{l}\text { Principal } \\
\text { functions }\end{array}$ \\
\hline \multirow[t]{2}{*}{1} & 2 & 3.51 & EmAgB1 & 000381200 & - & $\begin{array}{l}\text { Lipid binding, } \\
\text { Th2 response }\end{array}$ \\
\hline & $2-11,18$ & 44.68 & $\mathrm{EgAgB} 1$ & 000381200 & - & \\
\hline \multirow[t]{2}{*}{2} & 2,3 & 11.55 & EmAgB2 & 000381100 & - & $\begin{array}{l}\text { Lipid binding, } \\
\text { Th2 response }\end{array}$ \\
\hline & $2-10,13,15$ & 16.11 & $\operatorname{EgAgB} 2$ & 000381100 & - & \\
\hline 3 & $2-4$ & 29.49 & EmAgB3a & 000381500 & - & $\begin{array}{l}\text { Lipid binding, } \\
\text { Th2 response }\end{array}$ \\
\hline \multirow[t]{2}{*}{4} & $1-4$ & 27.34 & $\mathrm{EmAgB} 3 \mathrm{~b} / \mathrm{c}$ & $000381600 / 700$ & - & Lipid binding, \\
\hline & $2-6,9$ & 12.86 & EgAgB3 & 000381600 & - & Th2 response \\
\hline \multirow[t]{2}{*}{5} & $2-4$ & 5.67 & EmAgB4 & 000381400 & - & $\begin{array}{l}\text { Lipid binding, } \\
\text { Th2 response }\end{array}$ \\
\hline & $2-12,14,16,18$ & 54.24 & EgAgB4 & 000381400 & - & \\
\hline \multirow[t]{2}{*}{6} & 22 & 0.08 & Collagen $\alpha 1$ (IV) & 000140000 & P02463 & ECM \\
\hline & 22 & 0.08 & & 000144350 & & \\
\hline \multirow[t]{2}{*}{7} & 6,15 & 0.13 & GP5 & 000184900 & P21845 & $\begin{array}{l}\text { Serine protease, } \\
\text { Th2 response }\end{array}$ \\
\hline & $\begin{array}{c}5,6,10,11 \\
13,14,16,18\end{array}$ & 20.24 & & 000184900 & & \\
\hline \multirow[t]{2}{*}{8} & 9,10 & 0.22 & Cystatin & 000849600 & P21460 & $\begin{array}{l}\text { Protease } \\
\text { inhibitor }\end{array}$ \\
\hline & 9,10 & 1.36 & & 000849600 & & \\
\hline \multirow[t]{2}{*}{9} & 10 & 0.19 & $\mathrm{MDH}$ & 000417100 & P14152 & $\begin{array}{l}\text { Carbohydrate } \\
\text { metabolism }\end{array}$ \\
\hline & 10 & 0.68 & & & & \\
\hline \multirow[t]{2}{*}{10} & 10,11 & 0.18 & GAPDH & 000254600 & Q64467 & $\begin{array}{l}\text { Carbohydrate } \\
\text { metabolism }\end{array}$ \\
\hline & 10 & 0.55 & & 000254600 & & \\
\hline \multirow[t]{2}{*}{11} & 11,12 & 0.57 & FBA & 000905600 & Q91Y97 & $\begin{array}{l}\text { Carbohydrate } \\
\text { metabolism }\end{array}$ \\
\hline & $10-12$ & 1.86 & & 000905600 & & \\
\hline \multirow[t]{2}{*}{12} & 11,12 & 0.44 & Cathepsin B & 000790200 & Q923E4 & Cysteine protease \\
\hline & 11,12 & 1.69 & & 000790200 & & \\
\hline \multirow[t]{2}{*}{13} & $11,12,19$ & 0.1 & Laminin & 000068100 & Q61292 & ECM \\
\hline & 22 & 0.13 & & 000068100 & & \\
\hline \multirow[t]{2}{*}{14} & 13 & 0.5 & Enolase & 000514200 & P17182 & $\begin{array}{l}\text { Carbohydrate } \\
\text { metabolism }\end{array}$ \\
\hline & 12 & 0.41 & & 000514200 & & \\
\hline \multirow[t]{2}{*}{15} & 14 & 0.52 & EP45 & 000824000 & O08797 & Cysteine protease \\
\hline & 12 & 0.54 & & 000824000 & & \\
\hline
\end{tabular}

(Continued) 


\begin{tabular}{|c|c|c|c|c|c|c|}
\hline $\begin{array}{l}\text { Serial } \\
\text { No. }\end{array}$ & Band nos. & Sum of emPAI ${ }^{1}$ & Description & $\begin{array}{c}\text { Accession no. } \\
\text { (EmuJ_/EgrG_) }\end{array}$ & $\begin{array}{c}\begin{array}{c}\text { Mouse } \\
\text { homolog }^{3}\end{array} \\
\end{array}$ & $\begin{array}{l}\text { Principal } \\
\text { functions }\end{array}$ \\
\hline \multirow[t]{2}{*}{16} & 16 & 0.14 & GCP & 000824400 & Q9JK48 & Binding activity \\
\hline & 15,17 & 1.07 & & 000824400 & & \\
\hline \multirow[t]{2}{*}{17} & 18 & 0.63 & GCP & 000712600 & Q9JK48 & Binding activity \\
\hline & 20,21 & 1.89 & & 000712600 & & \\
\hline \multirow[t]{2}{*}{18} & 19 & 0.03 & $\alpha$ glucosidase & 000141000 & P70699 & $\begin{array}{l}\text { Carbohydrate } \\
\text { metabolism }\end{array}$ \\
\hline & 21 & 0.1 & & 000143500 & & \\
\hline \multirow[t]{2}{*}{19} & $17,21,22$ & 0.25 & HSPG & 000575900 & Q05793 & $\begin{array}{l}\text { Binding } \\
\text { activity }\end{array}$ \\
\hline & 21 & 0.31 & & 000575900 & & \\
\hline \multirow[t]{2}{*}{20} & 18 & 0.08 & $\mathrm{AAO}$ & 000530400 & Q8JZQ5 & Oxidoreductase \\
\hline & 17,19 & 0.39 & & 000530400 & & \\
\hline \multirow[t]{2}{*}{21} & 18 & 0.04 & ADAMTS3 & 000969100 & E9Q287 & Protease \\
\hline & 17,18 & 0.47 & & 000969100 & & \\
\hline \multirow[t]{2}{*}{22} & $18,21,22$ & 0.12 & Prosaposin & 000733100 & Q61207 & $\begin{array}{l}\text { Signaling, } \\
\text { growth }\end{array}$ \\
\hline & 21 & 0.15 & & 000733100 & & \\
\hline \multirow[t]{2}{*}{23} & 21 & 0.07 & $\alpha$ mannosidase & 000704400 & O09159 & $\begin{array}{l}\text { Carbohydrate } \\
\text { metabolism }\end{array}$ \\
\hline & 18 & 0.37 & & 000704400 & & \\
\hline \multirow[t]{2}{*}{24} & 22 & 0.04 & Peroxidasin & 000733600 & Q3UQ28 & Peroxidase \\
\hline & 21 & 0.09 & & 000733600 & & \\
\hline \multirow[t]{2}{*}{25} & 22 & 0.06 & Emb9 & 000139900 & P09055 & Binding activity \\
\hline & 22 & 0.08 & & 000144400 & & \\
\hline \multirow[t]{2}{*}{26} & 22 & 0.11 & Notch & 000343000 & Q01705 & Binding activity \\
\hline & 22 & 0.2 & & 000343000 & & \\
\hline \multirow[t]{2}{*}{27} & 22 & 0.11 & EGF & 000255800 & E9Q2T3 & Adhesion \\
\hline & 22 & 0.23 & & 000255800 & & \\
\hline \multirow[t]{2}{*}{28} & 15 & 0.08 & PGI & 000626300 & P06745 & $\begin{array}{l}\text { Carbohydrate } \\
\text { metabolism }\end{array}$ \\
\hline & 15 & 0.68 & & 000626300 & & \\
\hline
\end{tabular}

${ }^{1}$ Exponentially modified protein abundance index.

${ }^{2}$ Accession numbers were obtained from NCBInr DB (http://www.ncbi.nlm.nih.gov/) and E. multilocularis and

E. granulosus DB (http://www.genedb.org/Homepage/).

${ }^{3}$ Mouse homologs were retrieved using Uniprot software (http://www.uniprot.org/).

AAO, amiloride sensitive amine oxidase; ADAMTS3, ADAMTS protein 3; EgAgB, E. granulosus antigen B; EmAgB, E. multilocularis antigen B; Emb9, abnormal EMBroylocus tagsis emb 9; EP45, estrogen regulated protein EP45;

FBA, fructose bisphosphate aldolase; GAPDH, glyceraldehyde 3-phosphate dehydrogenase; GCP, gynecophoral canal protein; GP5, glycoprotein antigen 5; HSPG, basement membrane specific heparan sulfate; MDH, malate dehydrogenase (cytosolic); PGI, phosphoglucose isomerase. 
Table 4: Functional categorization of interacting proteins identified in EgHF and EmHF

\begin{tabular}{lcc}
\hline Interaction properties & No. of proteins found in EgHF & No. proteins of identified in EmHF \\
\hline Carbohydrate metabolism & 27 & 7 \\
Adhesion and cytogenesis & 18 & 20 \\
\hline
\end{tabular}

comparatively compiled proteome profile for $\mathrm{AE}$ and CE HF. The HFs shared biochemical and biological similarities. We identified 120 and 153 proteins from fully matured $\mathrm{AE}$ and $\mathrm{CE} \mathrm{HF}$, respectively, in which 56 and 84 proteins represented distinctive species. Those proteins were largely parasite proteins (44 and 66 molecules); hostderived proteins constituted minor fractions (12 and 18 molecules).

$\mathrm{AE}$ and $\mathrm{CE}$ metacestodes develop in intermediate hosts with different growth rates and morphological characteristics. The AE metacestode grows more rapidly than the E. granulosus metacestode. Morphologically, the AE metacestode typically forms a multilocular vesicular mass, while the $\mathrm{CE}$ metacestode matures into a large unilocular cyst [1]. The major proteins detected in those HFs and their functional PPI networks suggest both common and differing biological activity specific to the respective parasites' characteristic modes of maturation within hosts.

Diverse antigen $\mathrm{B}$ isoforms were widely distributed in both fluids. In EmHF, EmAgB3 constituted the most enriched population, followed by EmAgB2, EmAgB4, and $\mathrm{EmAgB}$. Conversely, the fractions of $\mathrm{EgAgB}$ isoforms were reversed, with major fractions of $\mathrm{EgAgB} 1$ and $\mathrm{EgAgB} 4$ and a minor EgAgB3 fraction (Tables 1 and 2, Figure 4). We could not detect EmAgB5 or EgAgB5 from fully mature EmHF or CE2 HF. Expression of these molecules might be associated with premature adult stages [21]. Antigen B proteins are multifunctional lipoproteins that inhibit neutrophil chemotaxis and dendritic cell maturation and the induction of host immune responses $[14,22,23]$. These molecules also function as hydrophobicligand binding proteins [15] and constitute the major components to supply energy and resources for maintaining cellular viability by providing essential host lipids [24] because cestode parasites lack the enzymes involved in lipid biosynthesis $[7,8]$. The dissimilar expression patterns of several antigen B molecules, which revealed interspecies differences, suggest their idiosyncratic roles in host-parasite specific interactions and pathobiological changes during the course of disease progression [11, 15].

The second most abundant proteins were carbohydrate-metabolizing enzymes. These molecules did not bear signal peptide, but significant amounts appeared to be secreted. The enzymes were commonly found in both fluids, and some additional molecules were detected in the EgHF. Carbohydrate-metabolizing enzymes are repeatedly observed in the secretome of several helminths including E. granulosus $[11,13]$. We thought that these molecules might be excreted through specific mechanism, i.e., extracellular vesicle mediated secretion rather than by passive leakage via tegumental shedding and/or turnover $[25,26]$. Previous studies indicated that some carbohydrate-metabolizing enzymes exerted moonlighting effects, such as the antioxidant detoxification and glutathione redox cycle, cellular adhesion and invasion, plasmin-mediate proteolysis, and IgA immune responses, together with their primary biochemical roles [27-30]. More enzymes in the EgHF might be correlated with longer survival of $\mathrm{CE}$ than $\mathrm{AE}$ owing to the more sufficient energy sources and by protecting the parasite from host defensive system.

Proteolytic enzymes and their inhibitors play major roles in numerous cellular events [31,32]. We detected relatively large amounts of these molecules, which strongly suggest that vigorous molecular biochemical and cell biological events operate dynamically in HFs. It is notable that we detected a great deal of NAALAD2 in EmHF. Upregulation of NAALAD2 expression has been associated with exacerbation of cancerous lesions and facilitation of tumor invasion [33]. Interestingly, we detected active NAALAD2 transcription only in the early developmental stages, one to three months, but not later than six month postinfection (Supplementary Figure 1). We also did not detect the NAALAD2 transcript in the CE2 stage. The considerable amounts of NAALAD2 observed in fully mature EmHF indicated the continuous accumulation of previously synthesized molecules (Table 2 ). This result suggests that infiltrative growth of $\mathrm{AE}$ vesicle might be associated with early but not late stage of infection. In the late stage, AE vesicle might expand by the inward growth of numerous daughter vesicles containing protoscolices, similar to the expansion of $\mathrm{CE}$ cyst. NAALAD2 protein accumulated in the EmHF might contribute to peripheral infiltrative growth to some extent. At the same time, highly crowded daughter vesicles increased unfavorable anoxic internal microenvironments and nutritional imbalance, which might result in the characteristic AE pathology of central necrosis. Further studies will be needed to gain deeper insight into the functional relevance and relationships between transcriptional activity and proteolytic activity, and infiltrative growth of $\mathrm{AE}$ vesicle.

Both HFs harbored some molecules closely related to developmental processes, i.e., notch, prosaposin, emb 9, hedgehog, and EGF domain proteins. They might participate in differentiation of neoblasts or stem cells during early embryogenesis and protoscolex maturation $[34,35]$. We also detected tissue connection and membrane structural proteins, such as different types of collagen 
chains, laminin, integrin, and HSPG (Figure 4). These ECM-associated molecules might exert their effects during adhesion, cytogenesis, and formation of cellular architecture through interactions with other molecules [36].

Host proteins comprised approximately $10 \%$ of the total proteins; this proportion was similar to that of Taenia solium metacestode cyst fluid [37]. Many of the host proteins detected in both HFs were serum proteins, strongly supporting the notion that Echinococcus might take up and exploit these substances. Other host molecules that function in the immune modulation and protective activity found in HF might also be absorbed through the highly permeable germinal layer during host-parasite interactions $[12,38]$.
We either did not detect some previously characterized proteins whose functions might be relevant to parasite homeostasis or they were exclusive to only one sample. For example, we observed membrane-sensing endophilin B1 (previously known as P29) and signalrelated 14-3-3 proteins only in EgHF (Figure 4 and Table 2). However, the authentic activity of those molecules was confirmed in E. multilocularis [39, 40]. This apparent discrepancy could suggest that expression levels of these molecules in E. multilocularis metacestode might not be sufficiently high to have been detectable under our experimental conditions. The use of different technical systems might also explain the detection of different protein repertories. Alternatively, the expression patterns of these

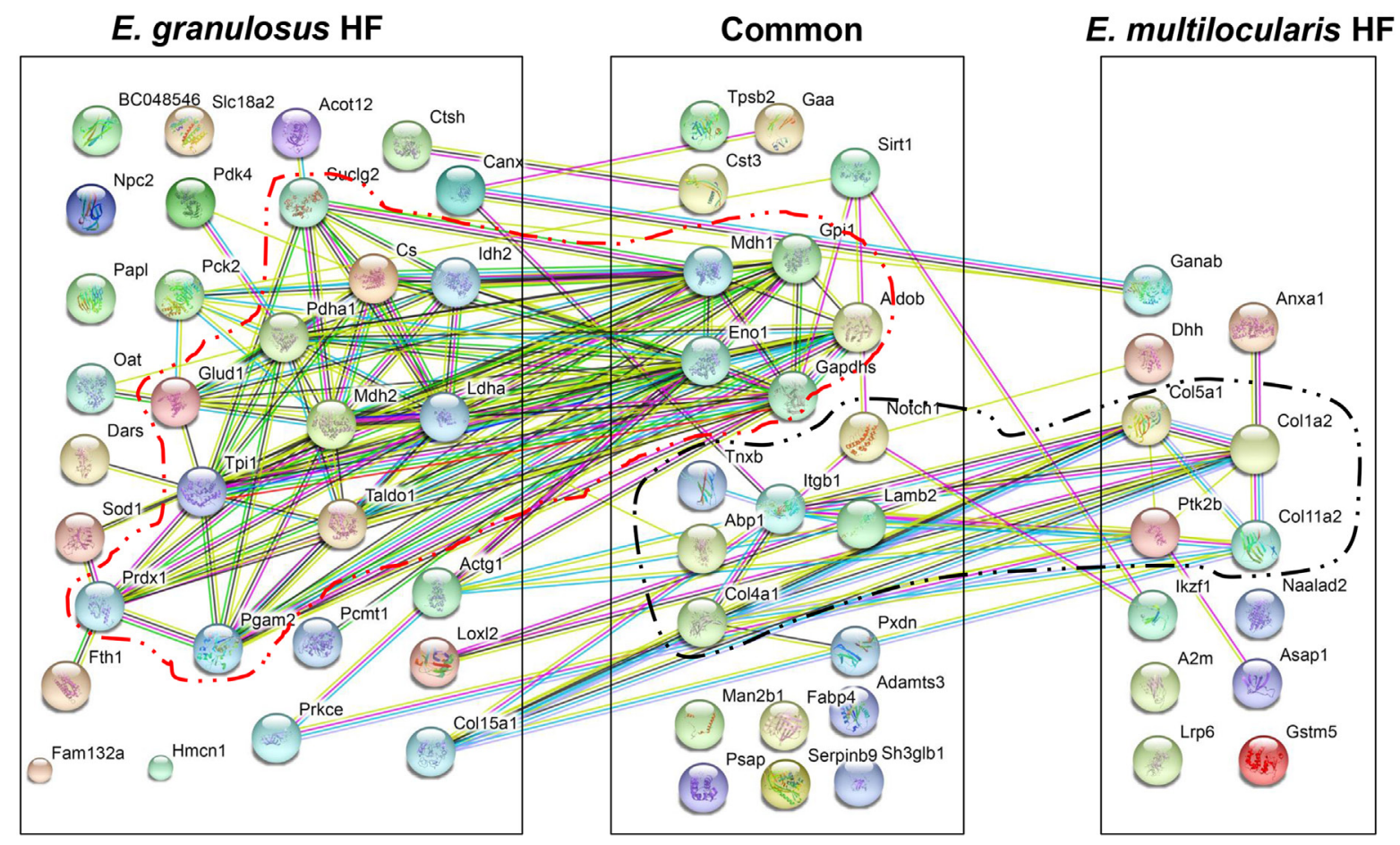

Figure 5: Protein-protein interaction networks in E. multilocularis hydatid fluid (EmHF) and E. granulosus HF (EgHF). The correlated interactions extracted from the EmHF and EgHF proteins are presented with their predicted functional partners by STRING ver10.0 (http://string-db.org/). The interactions are shown in confidence view. Proteins marked within lines represent a robust association. A2m, $\alpha 2$ macroglobulin; Abp1, amiloride sensitive amine oxidase; Acot12, acyl-CoA thioesterase 12; Actg1, $\gamma$ actin (cytoplasmic 1); Adamts3, ADAM metallopeptidase; Aldob, fructose-bisphosphate aldolase B; Anxa1, annexin A1; Asap1, ankyrin repeat and PH domain1; BC048546, cDNA sequence BC048546; Canx, calnexin; Col11a2, collagen type XI $\alpha 2$; Col15a1, collagen type XV $\alpha 1$; Col1a2, fibrillary collagen type I $\alpha 2$; Col4a1, collagen type IV $\alpha 1$; Col5a1, collagen type V $\alpha 1$; Cs, citrate synthase; Cst3, cystatin C; Ctsh, cathepsin H; Dars, aspartyl-tRNA synthetase; Dhh, desert hedgehog; Eno1, enolase 1; Fabp2, fatty acid binding protein 2; Fam132a, family with sequence similarity 132 (member A); Fth1, ferritin heavy chain 1; Gaa, $\alpha$-glucosidase; Ganab, glucosidase $2 \alpha$ neutral subunit; Gapdhs, glyceraldehyde-3-phosphate dehydrogenase, Glud1, glutamate dehydrogenase 1; Gpi1, glucose phosphate isomerase 1; Gstm5, glutathione transferase (mu-class); Hmcn1, hemicentin 1; Idh2, isocitrate dehydrogenase 2; Ikzf1, zinc finger 1; Itgb1, integrin $\beta 1$; Lamb2, laminin $\beta 2$; Ldha, lactate dehydrogenase A; Lox12, lysyl oxidase 2; Lrp6, low density lipoprotein receptor-related protein 6; Man2b1, mannosidase 2 $\alpha \mathrm{B} 1$; Mdh1, malate dehydrogenase 1; Mdh2, malate dehydrogenase 2 (mitochondrial); Naalad2, N-acetylated $\alpha$-linked acidic dipeptidase 2; Notch1, neurogenic locus notch protein; Npc2, Niemann Pick (C2); Oat, ornithine aminotransferase; Papl, RIKEN cDNA C330005M16; Pck2, phosphoenolpyruvate carboxykinase 2 (mitochondrial); Pcmt1, isoaspartate o methyltransferase 1; Pdha1, pyruvate dehydrogenase E1 a1; Pdk4, pyruvate dehydrogenase kinase; Pgam2, phosphoglycerate mutase 2; Prdx1, peroxiredoxin 1; Prkce, protein kinase C; Psap, prosaposin; Ptk2b, protein tyrosine kinase 2; Pxdn, peroxidasin; Serpinb9, serpin family B member 9 protease inhibitor; Sh3glb1, SH3 domain containing GRB2-like endophilin B1; Sirt1, NAD-dependent deacetylase sirtuin-1; Slc18a2, solute carrier family 18 (vesicular monoamine); Sod1, superoxide dismutase 1; Suclg2, succinate-CoA ligase (GDP-forming) subunit $\beta$ (mitochondrial); Taldo1, transaldolase 1; Tnxb, tenascin XB; Tpi1, triosephosphate isomerase 1; Tpsb2, tryptase $\beta 2$. 
proteins might be differentially regulated in different host conditions. A previous study demonstrated that AE vesicles grown in immunocompetent mice expressed significantly low levels of 14-3-3 protein compared with those grown in immune deficient nude mice [41].

Protein-protein interaction networks may provide valuable information on integrated protein functions [42]. One major PPI network was evident in each EmHF and EgHF, which might be correlated with survival strategies of these parasites (Figure 5). In EmHF, interactions among ECM-associated molecules revealed a symbiotic cluster. The hub proteins (integrin, laminin, and protein kinase 2) mediate cellular adhesion and cytogenesis, which might reflect morphological features characteristic of AE. AE might express more ECM-associated molecules to construct and maintain the highly complicated multilocular architecture [1]. The main PPI network found in EgHF was related to carbohydrate metabolic pathways in which lactate dehydrogenase, transaldolase 1 , and enolase 1 were the central proteins. Because CE cyst grows to be considerably large and shows longer lifespans than AE vesicle, enzymes and pathways involved in carbohydrate metabolism might be more active in CE cyst.

The main characteristics that differentiate these two phylogenetically closed parasite species are the modes of proliferation and invasion in the hosts. The protoscolex and germinal layer should have diverse bioactive molecules that exert their activity during the parasites' maturation. Therefore, a parallel proteomic analysis on the germinal layer and the protoscolex would provide more direct evidence of their pathogenesis. This should form the basis for future investigations.

In conclusion, this study comparatively analyzed the LC-MS/MS based proteome profiles of fully mature HF from E. multilocularis and E. granulosus metacestodes grown in immunocompetent hosts. We identified 120 and 153 proteins, respectively, from the EmHF and EgHF, most of which had parasitic origins. The major parasite protein fractions including multifunctional antigen B isoforms, carbohydrate-metabolizing enzymes, proteolytic enzymes and inhibitors, and ECM molecules were either common to both HFs or exclusive to one sample. Functional PPI networks established for the first time indicated that at least each one of major network might operate in both EmHF (symbiotic interactome relationship between EMC molecules) and EgHF (carbohydrate metabolism), which might reflect similar but different biological behaviors during the worm's maturation. Our results also highlight future strategies for developing chemotherapeutic and vaccine modalities against fatal chronic communicable diseases. Blocking antigen $\mathrm{B}$ isoforms may impede the uptake and transport of essential host lipids, which could significantly affect parasite survival. Disrupting key bioactive molecules involved in carbohydrate metabolism and ECM interactions may also be useful for novel interventions.

\section{MATERIALS AND METHODS}

\section{Parasites and sample preparation}

Kunming mice were each infected intraperitoneally with 1000 viable E. multilocularis protoscolices (EmRUS12 strain) [43] collected from naturally infected voles (Microtus fuscus) in an AE endemic area (Dari County, Qinghai Province, China) [24]. Metacestode vesicles grown for 9 months were harvested from the liver surface and peritoneal cavity. The vesicles were carefully decorticated and washed more than 10 times with physiological saline. The EmHF was aseptically drawn from individual intact cysts using a 26-gauge needle and were pooled in the presence of protease inhibitor cocktail (25 ml per tablet; Roche, Mannheim, Germany). In addition, AE masses were chronologically collected from the mice at $1,3,6,9$, and 14 months postinfection and were immediately used for RNA extraction using a commercially available kit (iNtRON, Seongnam, Korea). E. granulosus metacestode cyst (sensu stricto, G1 genotype) was obtained from a naturally infected sheep in a local abattoir in Xining (Qinghai Province, China). EgHF was drawn from a single fertile hepatic CE2 cyst [11]. The germinal layer and protoscoleces were used for RNA preparation. Both HFs were centrifuged at $500 \mathrm{~g}$ for $2 \mathrm{~min}$, followed by $20000 \mathrm{~g}$ for $1 \mathrm{~h}$. Supernatants (EmHF and EgHF) were dialyzed against phosphate buffered saline (PBS, $100 \mathrm{mM}, \mathrm{pH} 7.4$ ) for $4 \mathrm{~h}$, concentrated by lyophilization, and stored at $-80^{\circ} \mathrm{C}$. All procedures during the worm collection and $\mathrm{HF}$ dialysis were done at $4^{\circ} \mathrm{C}$ unless otherwise specified.

All protocols including parasite infection, recovery of the worms under intraperitoneal injection of $500 \mu \mathrm{l}$ of a $7: 3$ mixture of alfaxlone $(10 \mathrm{mg} / \mathrm{ml})$ and xylazine (23.32 $\mathrm{mg} / \mathrm{ml}$ ) and euthanasia of the moribund mice were approved by the Institutional Review Committee of the Qinghai Province Institute for Endemic Disease Prevention and Control (protocol 2013-7-22).

\section{Protein identification}

EmHF and EgHF (each $20 \mu \mathrm{g}$ ) were separated by Tricine SDS-PAGE (10\%) under reducing conditions. The gels were stained with Coomassie Brilliant blue G-250 (CBB). Protein bands were divided into 22 gel pieces along with their $\mathrm{CBB}$-stained banding patterns by their molecular weights and were sliced into fragments. Disulfide bonds were reduced with $10 \mathrm{mM}$ dithiothreitol. The proteins were alkylated with iodoacetamide (10 $\mathrm{mg} / \mathrm{ml}$ ) in $100 \mathrm{mM} \mathrm{NH} \mathrm{HCO}_{3}$. After dehydration and rehydration, the proteins were trypsin-digested (12.5 $\mathrm{ng} / \mu \mathrm{l})$ and the peptides were extracted from the gel pieces using a 50\% acetonitrile solution that contained $5 \%$ formic acid. All chemicals were purchased from Sigma-Aldrich (St. Louis, MO). The peptides were dried in a MIVAC DUO vacuum evaporator (Genevac, 
Ipswich, UK) and dissolved in deionized distilled water/ acetonitrile/trifluoroacetic acid (93:5:2). The samples were analyzed using nano-liquid chromatography-electrospray ionization-multi-stage mass spectrometry (LC-ESI-MS/ MS) employing a model 1200 nano-flow system (Agilent Technologies, Palo Alto, CA) connected to a LTQ linear ion trap mass spectrometer (Thermo Electron, San Jose, CA). The reversed-phase capillary column was $12 \mathrm{~cm}$ in length, $75 \mathrm{~mm}$ in inner diameter and in-house packed with $5 \mu \mathrm{m} 200 \AA$ A pore sized Magic C18AQ beads (Michrome BioResources, Auburn, CA).

The peptides were eluted in a linear gradient from 10 to $40 \%$ acetonitrile over $65 \mathrm{~min}$. MS survey was scanned for $300-2000 \mathrm{~m} / \mathrm{z}$ with three data-dependent MS/MS scans of isolation width of $1.5 \mathrm{~m} / \mathrm{z}$, normalized collision energy of $25 \%$, and dynamic exclusion duration of $180 \mathrm{sec}$. MS data were generated in RAW file format (Thermo Scientific) using the Xcalibur1.4 with Tune1.0. Peptide peaks were introduced into MS/MS ions search in the Mascot server (http://www.matrixscience.com). Mass values were selected with monoisotopic masses. Peptide and MS/MS tolerances were \pm 1.2 and $\pm 0.6 \mathrm{Da}$, respectively. To validate $\mathrm{MS} / \mathrm{MS}$ results, Mascot files were loaded on Scaffold ver3.6 (Proteome Software, Portland, OR). Individual ions scores $>55$ were considered significant or extensive homology $(P<0.05)$. In order to verify potential ambiguity between parasite- and hostderived proteins, we searched at least two peptides that were specific to either parasite or host. The proteins ambiguously assigned were excluded. We considered cysteine carbamidomethylation and methionine oxidation during the analyses. We independently analyzed each of the duplicated biological samples. We performed MS database search with merged files from non-redundant and expressed sequence tags on the NCBI database (https:// www.ncbi.nlm.nih.gov/) and the E. granulosus and E. multilocularis gene and protein database of the Sanger Institute (http://www.genedb.org/Homepage).

Signal peptide and non-classical secretions were predicted by PSORT (http://psort.hgc.jp/), SignalP (http:// www.cbs.dtu.dk/services/SignalP/), and the SecretomeP 2.0 server (http://www.cbs.dtu.dk/). A protein with $P$ value of the D-score $\leq 0.05$ was considered to possess a signal peptide. Non-classical pathways were defined when NN score was $\geq 0.5$.

\section{Assignment of gene ontology (GO) terms}

Functional classification of GO terms for the parasite and host proteins was carried out using Blast2GO ver4.0.7 (http://www.blast2go.com) with default parameters (cutoff of 30 for homology annotation) [19] according to the BLASTp searches of the identified proteins against the SwissProt (http://web.expasy.org/docs/swiss-prot guideline.html) and NCBInr database. We subsequently conducted GO-mapping, InterProScan, and graphical analysis based on the BLASTp results. Histograms of biological process, molecular function, and cellular component were generated using the second level of the GO hierarchy.

\section{Analysis of functionally integrated protein- protein interaction (PPI) networks}

We searched the EmHF and EgHF protein sequences for mouse homologs using Uniprot software (http://www. uniprot.org/) to predict functional PPI relationships [42]. We individually screened the UniProtKB entry numbers of the mouse homologs with the terms "multiple protein" and "Mus musculus" (Taxid: 10090). Subsequently, we entered the retrieved mouse homologs into the STRING ver10.0 database (http://string-db.org/) [44] for in silico mapping of putative physical and functional associations of EmHF and EgHF proteins. The average local clustering coefficient was 0.556 and the PPI enrichment $P$-value was 0 . Each node and edge represented a protein and an interaction of pairwise proteins. We defined nodes with relatively large numbers of edges as hub proteins.

\section{Reverse-transcription PCR (RT-PCR)}

Total RNAs (400 ng each) extracted from the AE mass (1, 3, 6, 9, and 14 months postinfection) and a single CE2 cyst were used for amplification of E. multilocularis NAALAD2 (EmNAALAD2; EmuJ_000908900) with a gene-specific forward primer (AACTGGAGAGATGTCTAC) and a reverse primer (CAGTGAACCAAGAAGTCC). RT-PCR cycling consisted of $30 \mathrm{~min}$ at $45^{\circ} \mathrm{C}$ and $5 \mathrm{~min}$ at $95^{\circ} \mathrm{C}$, followed by 35 cycles of $1 \mathrm{~min}$ at $95^{\circ} \mathrm{C}, 45 \mathrm{sec}$ at $50^{\circ} \mathrm{C}, 3 \mathrm{~min}$ at $72^{\circ} \mathrm{C}$, and a final extension for $10 \mathrm{~min}$ at $72^{\circ} \mathrm{C}$. PCR products were separated by $1 \%$ agarose gel electrophoresis and visualized by ethidium bromide staining. EmActin (EmuJ_000407400) was amplified as a positive control with gene-specific primers: forward, TCGAAGCGTGGTATTCTC and reverse, TTGAGTGGTGCCTCAGTT.

\section{Author contributions}

Conceived and designed the experiment: YK, CSA. Performed the experiments: CSA, JGK, XH. Analyzed the data: YK, CSA, IK. Contributed reagents/materials/ analysis tools: JGK, IK, XH. Wrote the paper: YK, CSA.

\section{ACKNOWLEDGMENTS}

Dr. Joo-Ho Shin, Division of Pharmacology, Department of Molecular Cell Biology, Sungkyunkwan University School of Medicine, Korea, is greatly acknowledged for his help during construction of proteinprotein interaction networks. 


\section{CONFLICTS OF INTEREST} interests.

The authors declare that they have no competing

\section{FUNDING}

This work was supported by NRF2016R1A63A11931725.

\section{REFERENCES}

1. Eckert J, Deplazes P. Biological, epidemiological, and clinical aspects of echinococcosis, a zoonosis of increasing concern. Clin Microbiol Rev. 2004; 17:107-35.

2. Budke CM, Carabin H, Ndimubanzi PC, Nguyen H, Rainwater E, Dickey M, Bhattarai R, Zeziulin O, Qian MB. A systematic review of the literature on cystic echinococcosis frequency worldwide and its associated clinical manifestations. Am J Trop Med Hyg. 2013; 88:1011-27.

3. Yang GJ, Liu L, Zhu HR, Griffiths SM, Tanner M, Bergquist R, Utzinger J, Zhou XN. China's sustained drive to eliminate neglected tropical diseases. Lancet Infect Dis. 2014; 14:881-92.

4. Gottstein B, Stojkovic M, Vuitton DA, Millon L, Marcinkute A, Deplazes P. Threat of alveolar echinococcosis to public health-a challenge for Europe. Trends Parasitol. 2015; 31:407-12.

5. Brunetti E, Kern P, Vuitton DA, Writing Panel for the WHO-IWGE. Expert consensus for the diagnosis and treatment of cystic and alveolar echinococcosis in humans. Acta Trop. 2010; 114:1-16.

6. Jones A, Bray RA, Khalil LF. Keys to the cestode parasites of vertebrates. In: Khalil LF, Jones A, Bray RA, editors. Wallingford: CAB International; 1994: 1-2.

7. Olson PD, Zarowiecki M, Kiss F, Brehm K. Cestode genomics - progress and prospects for advancing basic and applied aspects of flatworm biology. Parasite Immunol. 2012; 34:130-50.

8. Tsai IJ, Zarowiecki M, Holroyd N, Garciarrubio A, Sanchez-Flores A, Brooks KL, Tracey A, Bobes RJ, Fragoso G, Sciutto E, Aslett M, Beasley H, Bennett HM, et al. The genomes of four tapeworm species reveal adaptations to parasitism. Nature. 2013; 496:57-63.

9. Kern P, Wen H, Sato N, Vuitton DA, Gruener B, Shao Y, Delabrousse E, Kratzer W, Bresson-Hadni S. WHO classification of alveolar echinococcosis: principles and application. Parasitol Int. 2006; 55:S283-7.

10. Sánchez F, Garcia J, March F, Cardeñosa N, Coll P, Muñoz C, Auladell C, Prats G. Ultrastructural localization of major hydatid fluid antigens in brood capsules and protoscoleces of Echinococcus granulosus of human origin. Parasite Immunol. 1993; 15:441-7.
11. Ahn CS, Han X, Bae YA, Ma X, Kim JT, Cai H, Yang HJ, Kang I, Wang H, Kong Y. Alteration of immunoproteome profile of Echinococcus granulosus hydatid fluid with progression of cystic echinococcosis. Parasit Vectors. 2015; $8: 10$.

12. Santos GB, Monteiro KM, da Silva ED, Battistella ME, Ferreira HB, Zaha A. Excretory/secretory products in the Echinococcus granulosus metacestode: is the intermediate host complacent with infection caused by the larval form of the parasite? Int J Parasitol. 2016; 46:843-56.

13. Cui SJ, Xu LL, Zhang T, Xu M, Yao J, Fang CY, Feng Z, Yang PY, Hu W, Liu F. Proteomic characterization of larval and adult developmental stages in Echinococcus granulosus reveals novel insight into host-parasite interactions. J Proteomics. 2013; 84:158-175.

14. Riganò R, Buttari B, Profumo E, Ortona E, Delunardo F, Margutti P, Mattei V, Teggi A, Sorice M, Siracusano A. Echinococcus granulosus antigen B impairs human dendritic cell differentiation and polarizes immature dendritic cell maturation towards a Th2 cell response. Infect Immun. 2007; 75:1667-78.

15. Ahn CS, Kim JG, Han X, Bae YA, Park WJ, Kang I, Wang $\mathrm{H}$, Kong Y. Biochemical characterization of Echinococcus multilocularis antigen B3 reveals insight into adaptation and maintenance of parasitic homeostasis at the host-parasite interface. J Proteome Res. 2017; 16:806-23.

16. Siracusano A, Marguttia P, Delunardo F, Profumoa E, Riganòa R, Buttari $\mathrm{B}$, Teggi A, Ortona E. Molecular cross-talk in host-parasite relationships: the intriguing immunomodulatory role of Echinococcus antigen B in cystic echinococcosis. Int J Parasitol. 2008; 38:1371-6.

17. Mejri N, Gottstein B. Echinococcus multilocularis metacestode metabolites contain a cysteine protease that digests eotaxin, a CC pro-inflammatory chemokine. Parasitol Res. 2009; 105:1253-60.

18. Bae YA, Yeom JS, Wang H, Kim SH, Ahn CS, Kim JT, Yang HJ, Kong Y. Taenia solium metacestode fasciclin-like protein is reactive with sera of chronic neurocysticercosis. Trop Med Int Health. 2014; 19:719-25.

19. Götz S, García-Gómez JM, Terol J, Williams TD, Nagaraj SH, Nueda MJ, Robles M, Talón M, Dopazo J, Conesa A. High-throughput functional annotation and data mining with the Blast2GO suite. Nucleic Acids Res. 2008; 36:3420-35.

20. Walker M, Baz A, Dematteis S, Stettler M, Gottstein B, Schaller J, Hemphill A. Isolation and characterization of a secretory component of Echinococcus multilocularis metacestodes potentially involved in modulating the hostparasite interface. Infect Immun. 2004; 72:527-36.

21. Zhang W, Li J, Jones MK, Zhang Z, Zhao L, Blair D, McManus DP. The Echinococcus granulosus antigen B gene family comprises at least 10 unique genes in five subclasses which are differentially expressed. PLoS Negl Trop Dis. 2014; 4:e784. 
22. Ahn CS, Cai H, Kim JG, Han X, Ma X, Bae YA, Yang HJ, Kang I, Wang H, Kong Y. An Echinococcus multilocularis antigen B3 proteoform that shows specific antibody responses to active-stage alveolar echinococcosis. J Clin Microbiol. 2015; 53:3310-7.

23. Shepherd JC, Aitken A, McManus DP. A protein secreted in vivo by Echinococcus granulosus inhibits elastase activity and neutrophil chemotaxis. Mol Biochem Parasitol. 1991; 44:81-90.

24. Lee EG, Kim SH, Bae YA, Chung JY, Suh M, Na BK, Kim TS, Kang I, Ma L, Kong Y. A hydrophobic ligand-binding protein of the Taenia solium metacestode mediates uptake of the host lipid: implication for the maintenance of parasitic cellular homeostasis. Proteomics. 2007; 7:4016-30.

25. Marcilla A, Trelis M, Cortés A, Sotillo J, Cantalapiedra F, Minguez MT, Valero ML, Sánchez del Pino MM, MuñozAntoli C, Toledo R, Bernal D. Extracellular vesicles from parasitic helminths contain specific excretory/secretory proteins and are internalized in intestinal host cells. PLoS One. 2012; 7:e45974.

26. Cwiklinski K, de la Torre-Escudero E, Trelis M, Bernal D, Dufresne PJ, Brennan GP, O'Neill S, Tort J, Paterson S, Marcilla A, Dalton JP, Robinson MW. The extracellular vesicles of the helminth pathogen, Fasciola hepatica: biogenesis pathways and cargo molecules involved in parasite pathogenesis. Mol Cell Proteomics. 2015; 14:3258-73.

27. Lorenzatto KR, Monteiro KM, Paredes R, Paludo GP, da Fonsêca MM, Galanti N, Zaha A, Ferreira HB. Fructosebisphosphate aldolase and enolase from Echinococcus granulosus: genes, expression patterns and protein interactions of two potential moonlighting proteins. Gene. 2012; 506:76-84.

28. Sahoo S, Murugavel S, Devi IK, Vedamurthy GV, Gupta SC, Singh BP, Joshi P. Glyceraldehyde-3-phosphate dehydrogenase of the parasitic nematode Haemonchus contortus to complement $\mathrm{C} 3$ and inhibits its activity. Parasite Immunol. 2013; 35:457-67.

29. Gómez-Arreaza A, Acosta H, Quiñones W, Concepción JL, Michels PA, Avilán L. Extracellular functions of glycolytic enzymes of parasites: unpredicted use of ancient proteins. Mol Biochem Parasitol. 2014; 193:75-81.

30. Penkov S, Kaptan D, Erkut C, Sarov M, Mende F, Kurzchalia TV. Integration of carbohydrate metabolism and redox state controls dauer larva formation in Caenorhabditis elegans. Nat Commun. 2015; 6:8060.

31. Chung JY, Bae YA, Na BK, Kong Y. Cysteine protease inhibitors as potential antiparasitic agents. Expert Opin Ther Pat. 2005; 15:995-1007.

32. Robinson MW, Menon R, Donnelly SM, Dalton JP, Ranganathan S. An integrated transcriptomics and proteomics analysis of the secretome of the helminth pathogen Fasciola hepatica: proteins associated with invasion and infection of the mammalian host. Mol Cell Proteomics. 2009; 8:1891-907.
33. Whitaker HC, Shiong LL, Kay JD, Grönberg H, Warren AY, Seipel A, Wiklund F, Thomas B, Wiklund P, Miller JL, Menon S, Ramos-Montoya A, Vowler SL, et al. N-acetylL-aspartyl-L-glutamate peptidase-like 2 is overexpressed in cancer and promotes a pro-migratory and pro-metastatic phenotype. Oncogene. 2014; 33:5274-87.

34. Carvelli L, Libin Y, Morales CR. Prosaposin: a protein with differential sorting and multiple functions. Histol Histopathol. 2015; 30:647-60.

35. Vazin T, Ashton RS, Conway A, Rode NA, Lee SM, Bravo V, Healy KE, Kane RS, Schaffer DV. The effect of multivalent sonic hedgehog on differentiation of human embryonic stem cells into dopaminergic and GABAergic neurons. Biomaterials. 2014; 35:941-8.

36. Kubota Y, Nagata K, Sugimoto A, Nishiwaki K. Tissue architecture in the Caenorhabditis elegans gonad depends on interactions among fibulin-1, type IV collagen and the ADAMTS extracellular protease. Genetics. 2012; 190:1379-88.

37. Victor B, Kanobana K, Gabriël S, Polman K, Deckers N, Dorny P, Deelder AM, Palmblad M. Proteomic analysis of Taenia solium metacestode excretion-secretion proteins. Proteomics. 2012; 12:1860-9.

38. Thompson RC. Biology and systematics of Echinococcus. Adv Parasitol. 2017; 95:65-109.

39. Siles-Lucasa M, Merli M, Mackenstedt U, Gottstein B. The Echinococcus multilocularis 14-3-3 protein protects mice against primary but not secondary alveolar echinococcosis. Vaccine. 2003; 21:431-9.

40. Ahn CS, Bae YA, Kim SH, Kim JG, Yu JR, Yang HJ, Eom KS, Wang H, Kang I, Yang Y, Kong Y. Spatiotemporal expression patterns and antibody reactivity of Taeniidae endophilin B1. J Clin Microbiol. 2016; 54:2553-62.

41. Gottstein B, Haag K, Walker M, Matsumoto J, Mejri N, Hemphill A. Molecular survival strategies of Echinococcus multilocularis in the murine host. Parasitol Int. 2006; 55:S45-9.

42. Wu J, Vallenius T, Ovaska K, Westermarck J, Mäkelä TP, Hautaniemi S. Integrated network analysis platform for protein-protein interactions. Nat Methods. 2009; 6:75-7.

43. Konyaev SV, Yanagida T, Nakao M, Ingovatova GM, Shoykhet YN, Bondarev AY, Odnokurtsev VA, Loskutova KS, Lukmanova GI, Dokuchaev NE, Spiridonov S, Alshinecky MV, Sivkova TN, et al. Genetic diversity of Echinococcus spp. in Russia. Parasitology. 2013; 140:1637-47.

44. Szklarczyk D, Franceschini A, Wyder S, Forslund K, Heller D, Huerta-Cepas J, Simonovic M, Roth A, Santos A, Tsafou KP, Kuhn M, Bork P, Jensen LJ, et al. STRING v10: protein-protein interaction networks, integrated over the tree of life. Nucleic Acids Res. 2015; 43:D447-52. 